\title{
La efectividad de las reformas laborales sobre el nivel de desempleo. Un análisis del contexto de México y Colombia para el período 2002-2012*
}

\section{Effectiveness of labor reforms on the unemployment rate.}

An analysis of the context of Mexico and Colombia for the period 2002-2012

Angie Lorena Pradilla Chaparro**

Recibido: 2 de mayo de 2016

Revisado: 23 de mayo de 2016

Aprobado: 1 de junio de 2016

* Este artículo es producto de la práctica en investigación desarrollada en el Centro de Investigaciones Económicas Louis Joseph Lebret, O. P. en el proyecto de investigación realizado en el interior del grupo de investigación Economía y Humanismo, de la Facultad de Economía. Se agradecen los comentarios efectuados por la docente investigadora Aline Bento Ambrosio. Cómo citar este artículo: Pradilla Chaparro, A. L. (2016). La efectividad de las reformas laborales sobre el nivel de desempleo. Un análisis del contexto de México y Colombia para el período 2002-2012. Revista CIFE, 18(28), 311-156. (DOI: http://dx.doi.org/10.15332/s0124-3551.2016.0028.06) 


\section{Resumen}

El desempleo es una de las variables más influyentes en el análisis de la política económica de los países, ya que incide en múltiples dimensiones que afectan la productividad, la estabilidad del entorno macroeconómico y la calidad de vida de la población. Ante ese panorama, el presente artículo recopila un estudio de las principales estrategias diseñadas por países como Colombia y México en el mejoramiento de las condiciones laborales, así como una comparación sobre el impacto de estos mecanismos en variables como el desempleo y el crecimiento económico. En ese orden de ideas, el objetivo de este trabajo consiste en describir las principales causas y los efectos de las reformas laborales aplicadas en México y Colombia durante el período de 2002 a 2012, todo lo anterior mediante la revisión de algunos estudios sobre el tema y de datos macroeconómicos como el desempleo y el PIB.

Palabras clave: Economía internacional, reforma laboral, desempleo.

Clasificación JEL: F01, F02, J20, J30, J60

\section{Abstract}

Unemployment is one of the most influential variables in the analysis of countries' economic policies, since it affects multiple dimensions that affect productivity, the stability of the macroeconomic environment and the quality of life of the population. Given this scenario, this article compiles a study of the main strategies designed by countries such as Colombia and Mexico in the improvement of working conditions, as well as a comparison on the impact of these mechanisms on variables such as unemployment and economic growth. In this context, the purpose of this paper is to describe the main causes and effects of the labor reforms implemented in Mexico and Colombia during the period from 2002 to 2012, all this by reviewing some studies on the subject and macroeconomic data such as unemployment and GDP.

Keywords: International economy, labor reform, unemployment.

Classification JEL: F01, F02, J20, J30, J60 


\section{Introducción}

Desde hace décadas, la evolución de las variables macroeconómicas ha permitido observar y determinar el crecimiento económico de los países. Su importancia radica en que las variables producto interno bruto (PIB), desempleo e inflación, entre otras, son las responsables de describir cómo es la situación de un país en términos de la actividad económica; además, gracias a ellas es posible realizar estimaciones y prever cómo se desarrollará la economía en el futuro, ya sea a largo, mediano o corto plazo. Cabe resaltar que para este análisis es necesario que haya un correcto manejo de los indicadores que reflejan el uso de las variables macroeconómicas.

Sin embargo, y pese a la importancia de las demás variables, el desempleo ha sido visto como una de las problemáticas más preocupantes para los países del mundo, pues es una realidad que afecta a todos en general. En ese orden de ideas, es posible afirmar que el desempleo es una de las variables más influyentes en el análisis de la política económica de los países, ya que incide en múltiples dimensiones que afectan la productividad, la estabilidad del entorno macroeconómico y la calidad de vida de la población.

En especial, el indicador de desempleo afecta de manera directa sobre los habitantes de un país, puesto que tanto la productividad como el crecimiento dependen específicamente de cómo se desarrolla el empleo y cómo impulsa la producción de bienes y servicios que se ofrecen en un país. Por lo tanto, cuando existen altas tasas de desempleo en un país, es muy probable que se vean afectados los ingresos, la demanda agregada y el sistema económico en general.

Para observar las condiciones de los individuos en términos del empleo, es necesario puntualizar en las relaciones entre los empleadores y los empleados. Dichas relaciones se refieren, entonces, al mercado laboral, que es, en primera medida, uno de los principales receptores de los cambios presentes en el desempleo, en las tasas de ocupación y los salarios. Es allí donde las reformas laborales empiezan a tener una connotación sobresaliente dentro de una economía, puesto que la creación de empleos de calidad y perdurables en el tiempo para todos o al menos la mayoría de los individuos debe ser propiciada por políticas o programas focalizados y que corrijan los desequilibrios en el mercado de trabajo.

La reforma laboral, siendo una iniciativa para modificar la legislación con el objetivo de flexibilizar el mercado laboral, asiste por lo general a la problemática del desempleo, por lo tanto, la creación de nuevos empleos debería ser su efecto final. En este orden de ideas, y dada la importancia de los efectos del desempleo en una economía, es pertinente hacer una comparación entre países como Colombia y México, ya que ambas naciones han pasado por situaciones relevantes respecto al desempleo que los han convertido en focos de información significativa para esta investigación. 
Por un lado, Colombia ha vivido crisis económicas significativas en los últimos 10 años, no obstante, ha presentado también las tasas más altas de crecimiento de los últimos 30 años. Sin embargo, en términos de desempleo, la evolución ha tendido a crecer más que a disminuir, pues alrededor del año 2003, Colombia había alcanzado su tasa más alta de desempleo, con un $16 \%$, y en los años siguientes disminuyó, pero de igual forma se mantuvo elevada. Por su parte, México ha tenido consecuencias tanto económicas como sociales debido a las crecientes tasas de desempleo, pues la economía informal, la migración, la pobreza y no menos importante, la decreciente integración de los ciudadanos a los sistemas de protección tanto de salud como de pensiones han aumentado.

Junto con el análisis de las reformas que han sido impuestas en los dos países para aliviar problemas en el mercado laboral -las cuales inciden directamente sobre el crecimiento económico de los dos países y, por ende, en el bienestar de sus habitantes-, se resolverá la siguiente pregunta: ¿cuáles han sido los efectos sobre el desempleo luego de la aplicación de reformas laborales en Colombia y México desde el año 2002 hasta 2012?

Para determinar la incidencia de las variables destacadas y sus relaciones con los conceptos fundamentales de la economía, el presente artículo se organiza de la siguiente manera: la primera parte está constituida por la presente introducción; en la segunda sección se realiza una descripción de las reformas impuestas para Colombia y México, junto con una contextualización de los conceptos más importantes; en el tercer acápite se indaga acerca de cuáles han sido las consecuencias sobre el nivel de desempleo en los países y cómo estas han afectado al crecimiento económico y el desempleo desde 2002 hasta 2012; por último, se analizan los resultados de las reformas en los países y se presentan las conclusiones de la investigación.

\section{Fundamentación teórica}

El mercado laboral es un escenario determinante para la consolidación de un óptimo proceso de producción y distribución de la actividad económica. Según Resico (2011), este tipo de mercado está constituido por un grupo de trabajadores y empleadores que conforman la demanda y oferta de trabajo. Desde el contexto de la economía social analizada por el autor, en este escenario laboral debe existir un equilibrio entre la necesidad por generar ganancias y la distribución equitativa de esos beneficios entre los trabajadores, lo cual es fundamental para la percepción de su ingreso y la calidad de vida que puedan llevar.

Cada país presenta características particulares que son producto de su transformación histórica, política, social y económica. A partir del estudio "El mercado de trabajo en Colombia", en este país el mercado laboral ha estado determinado por dinámicas de 
corto plazo, como la productividad, la demanda agregada, la oferta laboral, así como también por las alteraciones en el comportamiento de variables exógenas tales como los niveles de salario. Según dicha investigación, este mercado puede ser considerado heterogéneo debido a la "poca movilidad interna de la mano de obra e importantes diferencias en las tasas de desempleo, ocupación y participación entre ciudades" (Arango y Hamann, 2012).

En contraste, el mercado laboral en México ha sido analizado desde las relaciones que pueden establecerse entre los trabajadores y las políticas gubernamentales. Bensusán (2013) cita a Huber (2002) para describir que los mercados laborales en este país presentan particularidades tales como "niveles bajos de calificación, regulaciones de alto perfil, altas tasas de rotación laboral, un extendido sector informal y sindicatos politizados, pero con escasa presencia en el lugar de trabajo y poder de negociación”.

Es evidente que estos factores inciden sobre los niveles de desempleo que pueden presentar los países. Según el Banco de la República (2016), el desempleo es una situación en la que una parte de la población que está en edad de trabajar (mayores de 12 años) no tiene empleo a pesar de que esté dispuesta a ejercer algún tipo de trabajo.

"Entre las características típicas del desempleo hay que mencionar también aquellos elementos relacionados con aspectos microeconómicos. Tal es el caso de la falta de flexibilidad en las regulaciones del mercado laboral" (Resico, 2011). Todo lo anterior enfatiza en las modalidades de contratación que están condicionadas por las políticas laborales de cada país y por las relaciones que se entablan entre sindicatos y empleadores.

El comportamiento de los mercados laborales y la importancia que su dinámica ejerce sobre los niveles de desempleo afectan otras variables macroeconómicas, como la productividad, la cual corresponde a "la producción promedio por trabajador en un período de tiempo. Puede ser medida en volumen físico o en términos de valor (precio por volumen) de los bienes y servicios producidos" (Instituto Peruano de Economía, 2016).

Así mismo, la tasa de paro afecta directamente diversos rubros de la economía y el bienestar de la sociedad, ya que, por ejemplo, la población percibirá menores ingresos, lo que afectará directamente el consumo y con ello la satisfacción de las necesidades básicas e igualmente incidirá directamente sobre el déficit fiscal frente a un menor recaudo de impuestos y presentará una reducción de la producción real frente a un menor aporte de la población; todos estos factores incidirán negativamente sobre el crecimiento económico, el cual se puede definir como el aumento sostenido del producto en una economía, comúnmente medido por medio de variables económicas como el PIB, dejando de lado variables sociales (Álvarez y Alonso, 2005).

A raíz de todo lo anterior, es importante destacar que, con el objetivo de mejorar las condiciones socioeconómicas de la población e incidir positivamente sobre la actividad 
económica del país, los gobiernos recurren al uso de diversos macanismos, uno de ellos se centra en mejorar las condiciones laborales de los trabajadores y, a la vez, en buscar la disminución de la tasa de paro, de esta forma surge el concepto de reforma laboral. Al respecto, María Vega (2006) argumenta que una reforma laboral introduce modificaciones al Código de Trabajo que regula la relación laboral; se establece a partir de la premisa que plantea que todas las personas que pertenecen a la sociedad deben tener derecho al trabajo, es decir, que se deben buscar métodos que permitan disminuir las altas tasas de desempleo, así como también regular y proteger los derechos sociales de los trabajadores.

Otro aspecto que tiene en cuenta la reforma laboral es el fortalecimiento de rubros importantes tales como la productividad; esto se puede lograr por medio de una mayor capacitación laboral. De igual manera, busca fortalecer los ingresos de los trabajadores con el objetivo de disminuir la brecha de desigualdad socioeconómica existente en la sociedad. No obstante, en la búsqueda de la disminución de la tasa de desempleo, muchas veces las reformas laborales les otorgan a las empresas mayor flexibilización en el mercado laboral, lo que trae consigo efectos negativos para los trabajadores, ya que se presenta mayor facilidad para contratar y despedir empleados.

\subsection{Reforma laboral en Colombia}

Guataquí y García Suaza (2009) citan al Ministerio de Protección Social (2004) para describir que la reforma laboral en Colombia fue una idea que surgió con el objetivo de combatir los factores de origen que estaban determinando las altas tasas de desempleo. Las políticas estuvieron enfocadas en cuestiones de interés nacional como "recuperación del crecimiento económico, actualización de las normas laborales, fomento de iniciativa privada, fortalecimiento del sistema educativo y de capacitación y creación de mecanismos de protección a la población más vulnerable" (Guataquí y García Suaza, 2009).

Las principales razones que dieron pie a la realización de esta reforma se relacionan con la manifestación de grandes problemáticas que abarcan dimensiones sociales, económicas y jurídicas. Según el Boletín 74 de la Universidad EAFIT (2006), es viable deducir que el aspecto social se encontraba inmerso en un escenario de grandes dificultades en materia de educación, capacitación y acceso a sistemas de seguridad social, y en el cual la experiencia laboral de los jóvenes era mínima; pero desde el punto de vista jurídico, el contexto estaba determinado por la asimetría de la información, así como por el incumplimiento sobre las exigencias de los horarios laborales. En cuanto al componente económico, las altas tasas de despido y la profunda diferencia entre los salarios y los beneficios de las empresas contribuyeron a la discrepancia entre trabajadores y empleadores. 
Tabla1. Causas de la reforma laboral de Colombia para el año 2002

\begin{tabular}{|c|c|}
\hline Aspecto & Causas \\
\hline \multirow{7}{*}{ Social } & - Falta de acceso a derechos como salud, trabajo y pensión. \\
\hline & $\begin{array}{l}\text { - Alta deserción estudiantil por parte de los niños y jóvenes que componen las } \\
\text { familias de padres y madres cabeza de hogar, que se encontraban desempleados. }\end{array}$ \\
\hline & - Bajos niveles de mano de obra calificada. \\
\hline & • Desempleo de jóvenes. \\
\hline & - Bajo nivel de cobertura y poco alcance del Sistema de Seguridad Social. \\
\hline & $\begin{array}{l}\text { - Carencia de formación académica y de aptitudes laborales que demandan las } \\
\text { empresas. }\end{array}$ \\
\hline & $\begin{array}{l}\text { - No reconocimiento de las prácticas laborales de estudiantes y su debida } \\
\text { remuneración. }\end{array}$ \\
\hline \multirow{3}{*}{ Jurídico } & • Desconocimiento sobre las reglas en materia laboral por parte de los trabajadores. \\
\hline & - Mayor número de horas trabajadas que las permitidas. \\
\hline & - Proliferación del fenómeno de la informalidad. \\
\hline \multirow{4}{*}{ Económico } & $\begin{array}{l}\text { - Pérdida de ingreso por parte de los trabajadores y aumento de los beneficios de los } \\
\text { empresarios. }\end{array}$ \\
\hline & • Bajos salarios mínimos. \\
\hline & - Incremento superior del costo de vida frente a las remuneraciones. \\
\hline & - Altas tasas de despido de trabajadores próximos a cumplir 10 años de antigüedad. \\
\hline \multicolumn{2}{|r|}{ Fuente: elaboración propia con base en información de las instituciones de cada país. } \\
\hline \multicolumn{2}{|c|}{$\begin{array}{l}\text { La tabla } 1 \text { proyecta las diferentes razones que generaron la realización de una reforma } \\
\text { laboral en Colombia durante el año } 2002 \text {. Estas fuentes se encuentran clasificadas a } \\
\text { partir de criterios específicos tales como el aspecto social, el jurídico y el económico. } \\
\text { En ese orden de ideas, es importante indagar sobre los principales proyectos que se } \\
\text { llevaron a cabo en pro de mejorar el entorno laboral del país. De esa manera, se } \\
\text { mencionarán las reformas más destacadas dentro de los diferentes aspectos sociales, } \\
\text { jurídicos y económicos. }\end{array}$} \\
\hline
\end{tabular}

\section{Modificaciones sociales}

La aplicación de las reformas del año 2002 pretendía aportar al mejoramiento de todo escenario laboral, lo que incluía la cooperación para el fortalecimiento del desempleo de los jóvenes mediante la restructuración del contrato de aprendizaje, que incluye: 
Las prácticas con estudiantes universitarios, técnicos o tecnólogos [...] La realizada en las empresas por jóvenes que se encuentren cursando los dos últimos grados de educación lectiva $[\ldots]$ y el aprendiz alumno matriculado en los cursos dictados por el SENA. (Servicio Nacional de Aprendizaje - SENA, 2014).

Esta nueva visión proyecta un entorno diferente para la adquisición de experiencia, que es una exigencia dentro del mercado de trabajo.

En cuanto a la remuneración, "el apoyo del sostenimiento durante la fase práctica será equivalente al setenta y cinco por ciento $(75 \%)$ de un salario mínimo mensual legal vigente" (Congreso de la República de Colombia, 2002). Por ello, el apoyo a los estudiantes en proceso de formación es un incentivo para mantener el sostenimiento de la actividad laboral, lo que beneficia la expansión de la actividad económica del país en diferentes dimensiones, además, funciona como estímulo para la contratación de mano de obra calificada.

Añadido a lo anterior, como nuevo consenso en el contrato de aprendizaje se pretendía que este fuera "obligatorio para las empresas con más de quince empleados, pero su naturaleza deja de ser laboral, por lo que el trabajador no recibirá prestaciones, con excepción de los pagos a salud y riesgos profesionales" (Amarente, Arim y Santamaría, 2005). Esta situación es un impulso para la inclusión y el ensanchamiento del Sistema de Seguridad Social del país, pues reconoce la inserción de la nueva base de la población económicamente activa, mediante un proceso de cobertura.

Con estas reformas, se diseñaron mecanismos de intervención cuyo propósito fuera contrarrestar los efectos negativos que el aumento del desempleo deja a su paso. Uno de estos instrumentos fue el subsidio al empleo para la pequeña y mediana empresa, el cual se proyectaba como un medio temporal. Según el Boletín 74 de la Universidad EAFIT (2006), este iba a apoyar a "220 000 jefes de hogar desempleados, quienes [contarían] con un subsidio de hasta 100000 pesos mensuales durante un período de seis meses". Los recursos se iban a tomar de fuentes como el presupuesto nacional, transferencias hacia departamentos y créditos externos. Sin embargo, esto permite cuestionarse sobre los rubros a los que se les quitaría parte del suministro y el impacto que esto podría generar a largo plazo.

Es importante mencionar que la Ley 789 de 2002, que sustentaba esta reforma, también contemplaba las condiciones del contexto nacional, ya que reconoció que los subsidios también deberían estar orientados "en la asignación de recursos de las zonas rurales, en especial aquellas que presentan problemas de desplazamiento y conflicto campesino" (Universidad Externado Colombia, 2005).

Sumado a esto, el subsidio buscaba beneficiar a "las mujeres cabeza de hogar desempleadas temporalmente y con hijos menores de 18 años" (Universidad EAFIT, 2006). 
La razón central de este auxilio era fortalecer la asistencia de los niños y jóvenes a las instituciones de educación mediante la contratación de jefes de hogar que estuvieran desempleados, por lo cual, era necesrio que el acceso a este tipo de oportunidades de carácter laboral se articulara con otros programas diseñados en función de mejorar las condiciones sociales de toda la población.

La transformación del escenario laboral no solo busca reducir las altas tasas de desempleo, sino que también tiene como objetivo disminuir la informalidad. Para alcanzar sus propósitos, fue esencial organizar el Sistema de Protección Social, el cual consiste en aplicar políticas públicas que mejoren las condiciones de los individuos por medio de la garantía de derechos como la salud, la pensión y el trabajo.

Según la Ley 789 de 2002, el sistema debía prever las condiciones para que los trabajadores se adaptaran a los cambios que generarían las otras reformas, al mismo tiempo que se debían contemplar los cambios de la economía dinámica que presenta el país. En ese orden de ideas, uno de los principales aportes fue la "creación del Fondo de Protección Social [...] como una cuenta especial de la nación, sin personería jurídica, [...] cuyo objeto será la financiación de programas sociales que el Gobierno nacional defina como prioritarios" (Congreso de la República de Colombia, 2002) y aquellos proyectos que estén en función de la paz.

Dentro del análisis de esta estructura, el programa de pensiones y el acceso a la salud desempeñan un papel fundamental en las diferentes empresas y en los múltiples sectores de la economía, ya que funcionan como incentivos para formalizar diferentes puestos de trabajo y ofrecer las condiciones mínimas de empleo a los trabajadores.

Por otro lado, el crecimiento y el desarrollo económico del país exigen la formación de capital humano en diferentes campos. Este proceso incluye la inversión en investigación, el uso de nuevas tecnologías y, por lo tanto, la capacitación de la población. Se busca así incentivar el establecimiento de mano de obra calificada que contribuya a la producción de bienes con mayor valor agregado y también la consolidación de un sólido sector de servicios, aspectos que son determinantes para la participación del país en los mercados internacionales en términos de competitividad y costos de oportunidad.

Uno de los aspectos tratados en la reforma del año 2002, que buscaba aportar a esta situación, fue el proyecto de capacitación para inserción laboral, que se basaba en tomar de "las contribuciones parafiscales destinadas al Servicio Nacional de Aprendizaje [...] para la capacitación de población desempleada" (Congreso de la República de Colombia, 2002). Con este tipo de mecanismos, la reforma pretendía garantizar un puesto de trabajo que no solo generara ingresos a las familias, sino que también contribuyera a la formación educativa y a la expansión de oportunidades que les permitiera a los individuos ascender dentro de la esfera social. 
Según Macías, Suescún y Cardona (2009), este tipo de políticas suele focalizarse en una población objetivo, en este caso, los jóvenes. "Esta población es considerada prioritaria porque presenta los niveles más altos de desempleo y porque carece de habilidades básicas, psicosociales y capacitación demandadas por los segmentos modernos del mercado de trabajo" (Macías, Suescún y Cardona, 2009). Cabe señalar que la cobertura en empleo de esta parte de la población es una preocupación de interés nacional debido a que los jóvenes representan una base importante de la fuerza laboral del país. Por esa razón, es esencial invertir en el proceso de formación, cuyos resultados se deben interconectar con el empleo de otros factores, como la investigación y el uso de nuevas tecnologías.

Con la reforma laboral del año 2002 se crearon proyectos específicos tales como Jóvenes en Acción, que consiste en apoyar a "jóvenes en condición de pobreza y vulnerabilidad, con la entrega de transferencias monetarias, para que puedan terminar sus estudios técnicos, tecnológicos y profesionales" (Prosperidad Social, 2016).

Añadido a esto, también es necesario destacar la adopción de otros programas que se crearon posteriormente y que buscaban fortalecer la Ley 789 2002; entre estos se destacan Joven Competitivo (2003-2005), para el empleo sostenible, el cual se centró en la capacitación laboral y personal de jóvenes en Medellín. También sobresalió el programa Jóvenes con Futuro, que se enfocó en el desarrollo de "un proyecto de vida, competencias ciudadanas, nivelación de competencias, orientación sociolaboral y emprendimiento" (Macías, Suescún y Cardona, 2009).

\section{Modificaciones jurídicas}

La jornada laboral fue uno de los criterios que se tuvieron en cuenta en la reglamentación jurídica debido al fuerte impacto que genera sobre la relación entre el rendimiento del factor trabajo y la producción. Según Amarente, Arim y Santamaría (2005), con la reforma se realizaron modificaciones en "la jornada nocturna, que implica el pago de un recargo por parte del empleador [...] La nueva ley establece también la flexibilidad horaria [...]. Disminuye también el recargo pagado por el empleador los días domingo o festivos". Esta transformación ha sido fundamental para el sector terciario (comercio y servicios), según los autores, ya que contribuye al crecimiento de su producto y a la generación de nuevos puestos de trabajo.

Otra de las características que se relacionó con la modificación de la jornada laboral fue el establecimiento de nuevos acuerdos. A partir de la Ley 789 de 2002, "el empleador y el trabajador pueden acordar temporal o indefinidamente la organización de turnos de trabajo [...], podrán acordar que la jornada semanal de 48 horas se realice mediante jornadas diarias [...] distribuidas en máximo seis días a la semana" (Congreso de la República de Colombia, 2002). De esa manera, es posible deducir que este cambio traslada a otra posición el papel que desempeña el trabajador dentro del ambiente laboral, 
lo que conduce al reconocimiento de la importancia de su participación en el proceso de producción y en la prestación de servicios.

\section{Modificaciones económicas}

Uno de los propósitos más importantes de la reforma laboral del año 2002 fue modificar el manejo del costo de despido. "La reforma reduce la indemnización por cese unilateral de un contrato [a] término indefinido [...] Se intentó con esta medida evitar la elevada tasa de despido que se producía [de] trabajadores cercanos a cumplir los diez años de antigüedad" (Amarente, Arim y Santamaría, 2005). Según lo anterior, es posible inferir que el objetivo de esta iniciativa pretendía contribuir a la continuidad laboral, evitando la consolidación de fenómenos como el desempleo friccional, cuyo período afecta la productividad y la calidad de vida de los individuos al dejar de percibir su ingreso.

En síntesis, la reforma laboral sustentada en la Ley 789 de 2002 en Colombia propuso diferentes mecanismos de reactivación del mercado de trabajo con la finalidad de transformar el panorama económico y social del país. La mayoría de los programas mencionados tenía como eje común la expansión de la actividad productiva y el reconocimiento de los derechos de los trabajadores en términos de salud, pensiones y acceso a las oportunidades.

Es importante destacar que la flexibilización de la jornada laboral y el apoyo al desempleo juvenil suelen ser factores determinantes en las grandes economías, esencialmente a largo plazo, como base del sostenimiento de los sistemas de seguridad social. Añadido a lo anterior, el programa de capacitación brinda un nuevo escenario sobre la formación de capital humano y la utilidad que representa dentro del crecimiento y desarrollo económico del país.

\subsection{Reforma laboral en México}

Por otro lado, en cuanto a México, la reforma a la Ley Federal del Trabajo de 2012 ( Congreso de los Estados Unidos Mexicanos, 2012) ha sido la más importante a lo largo de la historia de este país, esto se debe a que la Secretaría de Gobernación de México (2013) establece que esta reforma implicó 363 modificaciones que buscaban mejorar las condiciones laborales de la sociedad, mayor integración a la seguridad social, disminución de las altas de paro y mayor formalidad, todo esto con el objetivo de incidir positivamente sobre la competitividad y productividad, con el fin de asegurar situaciones de índoles que impulsen el crecimiento económico.

Vale la pena destacar que surgieron principalmente a raíz de las crecientes tasas de desempleo acompañadas de un aumento de la economía informal, la migración, la pobreza y la decreciente integración de los ciudadanos a los sistemas de protección tanto de salud como de pensiones. 
Para analizar y explicar las principales modificaciones realizadas a la Ley Federal del Trabajo en México por medio de la reforma de 2012, en la tabla 2 se agrupan los aspectos económicos, sociales y jurídicos primordiales y se presentan las principales causas por las cuales se incorporó cada una de las modificaciones:

Tabla 2. Causas de la reforma a la Ley Federal del Trabajo de 2012

\begin{tabular}{|c|c|}
\hline Aspecto & Causas \\
\hline \multirow{9}{*}{ Social } & - Condiciones laborales precarias. \\
\hline & - Segmentación de grupos vulnerables. \\
\hline & - Explotación por trabajo infantil. \\
\hline & - Brecha de género laboral. \\
\hline & - Nula oferta laboral para los discapacitados. \\
\hline & $\begin{array}{l}\text { - Poca regulación en el trabajo doméstico, amplias jornadas experimentadas y bajos } \\
\text { salarios. }\end{array}$ \\
\hline & - Bajo bienestar para los trabajadores del campo. \\
\hline & - Sobreuso del régimen de subcontratación. \\
\hline & - Ineficiencia por parte de los sindicatos. \\
\hline \multirow[t]{6}{*}{ Jurídico } & $\begin{array}{l}\text { - Ineficiencia en los procesos judiciales, baja confianza de los empleados en este sector } \\
\text { y altos costos implícitos. }\end{array}$ \\
\hline & - Problemas con los salarios vencidos. \\
\hline & - Bajo consumo y poder adquisitivo de los trabajadores. \\
\hline & - Informalidad laboral. \\
\hline & - Baja productividad. \\
\hline & - Baja movilidad social. \\
\hline \multirow{3}{*}{ Económico } & - Capacitaciones ineficientes. \\
\hline & - Bajos salarios. \\
\hline & $\begin{array}{l}\text { - Infraestructura de establecimientos laborales peligrosa para el bienestar de los } \\
\text { empleados. }\end{array}$ \\
\hline
\end{tabular}

- Sanciones débiles.

- Poco incentivo a la utilización de servicios financieros. 


\section{Modificaciones sociales}

En primera instancia, José de Jesús González (2013) explica que, con el objetivo de mejorar las condiciones precarias de los trabajadores y garantizar en mayor medida sus derechos, se incorporó el concepto de "trabajo decente", recomendado por la Organización Internacional del Trabajo, que se enfoca en la dignidad laboral, la cual plantea un escenario en donde no exista discriminación y en el que todos los trabajadores tengan acceso a la seguridad social, acompañado de mejores salarios, mayor formación de capital humano por medio de las capacitaciones y derecho a la expresión. De esta manera, se observa que dicho concepto se añadió para fortalecer los derechos de los trabajadores al identificar al empleado como persona y resaltar la importancia de su dignidad.

En ese orden de ideas, y con el propósito de mejorar las condiciones de los trabajadores, se incluyeron medidas orientadas a la protección de diversos grupos frágiles que estaban siendo explotados laboralmente. En primera instancia, se destaca como delito y se ordena poner fin a la contratación de menores de 14 años fuera del círculo familiar (Soria, 2015), por lo tanto, el principal fin de este aspecto es reducir y eliminar el trabajo infantil. No obstante, la Secretaría de Gobernación de México (2013) plantea que, para los mayores de 14 años y menores de edad, se incorporó una serie de trabajos con riesgo que no podían realizar, pero cuya contratación no se estipuló como delito, por lo que es posible deducir que, aunque se buscó erradicar el empleo infantil, se siguió permitiendo la explotación laboral a los mayores de 14 años. Así mismo, para los mexicanos que laboran en otros países, se estableció una serie de modalidades de contratación que regulan la prestación de sus servicios (Gobierno de la República de México, 2013).

Por otro lado, según Jesuswaldo Soria (2015), frente a la creciente segregación y segmentación laboral en contra de la mujer, se generó mayor fortalecimiento de sus derechos laborales por medio de la prohibición de la discriminación por género mediante sanciones al acoso sexual, prohibición del requerimiento en algunas entidades y empresas de certificados de no embarazo para contratar, y se amplió asimismo la licencia de maternidad, en la que se le garantiza a la mujer un sueldo durante dicho período, todo esto con el fin de eliminar la creciente brecha de género laboral y promover el respeto por la mujer.

Otro grupo vulnerable que estaba siendo excluido, ya que contaba con una nula oferta laboral, era el de los discapacitados, por lo que se crearon medidas que permitieran ampliar la oferta laboral a aquellas personas con discapacidades físicas y se les exigió a empresas medianas y grandes acondicionar sus instalaciones para estas personas y promover así mismo su contratación (González, 2013).

En cuanto a los trabajadores domésticos, ante la creciente duración de las jornadas experimentadas y la poca regulación existente en este ámbito, se decidió aumentar la intervención en este campo y formalizar la duración de las jornadas y los pagos por parte 
de los empleadores. Por otro lado, a raíz de las nefastas condiciones experimentadas por los trabajadores de las minas, se formuló una serie de reglas y requerimientos para el desarrollo de sus actividades, de manera tal que se garantice mayor seguridad social (González, 2013). Por último, en lo que respecta a los trabajadores rurales, la Secretaría de Gobernación de México (2013) estableció el concepto de "trabajadores de campo", el cual obliga a los empleadores a implementar unas condiciones mínimas de trabajo que garanticen mayor bienestar por medio de seguros de vida, aumentos salariales y transportes a lugares de trabajo. Todas las modificaciones analizadas anteriormente se enfocan en aumentar la intervención en las condiciones laborales de diversos grupos vulnerables, con el objetivo de garantizarles un mayor bienestar y disminuir la tasa de paro.

Ahora bien, antes de la reforma se evidenciaba un aumento de la modalidad de contratación por prestación de servicios, esto quiere decir que los empleados no tenían formalmente un contrato ni acceso a la seguridad social, lo que facilitaba el despido de trabajadores y la minimización de costos. Marcela Calderón (2013) argumenta que, a raíz de dicha situación, mediante la reforma laboral de 2012 se reguló este régimen de subcontratación, ya que se buscaba mayor seguridad social por medio de la formalización de un contrato, por lo que este tipo de contratación se limitaría.

Por último, en cuanto al derecho de expresión laboral por medio de los sindicatos, se reorganizó su estructura y se estipuló un mecanismo de elección democrático (Secretaría de Gobernación de México, 2013), todo esto con el objetivo de promover mayor eficiencia en la prestación de sus servicios e igualdad para los miembros pertenecientes, resaltando valores como la imparcialidad, la equidad y el respeto.

\section{Modificaciones jurídicas}

Sumado a lo anterior, con el objetivo de aumentar la confianza de los empleados en el medio jurídico en aspectos relevantes como la disminución de costos en materia de tiempo y la repartición de justicia laboral, la cual se busca que sea eficiente, se modificó y modernizó la Ley Federal del Trabajo (Congreso de los Estados Unidos Mexicanos, 2012). Según la Secretaría de Gobernación de México (2013), para lograr dicho objetivo, se llevó a cabo la implementación de un servicio proveniente de la Junta Federal de Conciliación y Arbitraje que añade el principio de reconciliación en el ámbito laboral. Este principio permite utilizar medios tecnológicos en los juicios laborales para reducir costos en materia de tiempo y así mismo sancionar a los funcionarios que obstaculicen un juicio laboral. Este medio se utiliza principalmente en problemas relacionados con prestaciones sociales, aportaciones de vivienda y prestaciones del sistema de ahorro, todo esto en la búsqueda de mayor eficiencia y credibilidad por parte de los empleados al sistema jurídico. 


\section{Modificaciones económicas}

Las reformas laborales tienen por objetivo introducir reestructuraciones al Código de Trabajo con el fin de disminuir la tasa de paro y mejorar las condiciones sociales de los trabajadores. Por ello, la reforma a la Ley Federal del Trabajo de 2012 (Congreso de los Estados Unidos Mexicanos, 2012) se centró en los aspectos mencionados anteriormente y en la disminución de las altas tasas de informalidad, de manera que se centró en la población más vulnerable y se enfocó principalmente en mejorar la productividad y la competitividad del país, asegurando el impulso del crecimiento económico.

Para ello, según Jesuswaldo Soria (2015), agregó múltiples modificaciones en los mecanismos de contratación, así estimuló la creación de empleos principalmente a la población vulnerable, pero también se enfocó en las madres cabeza de familia, los jóvenes y los adultos mayores, brindándoles empleos orientados a su determinada situación; esto se vio acompañado del fortalecimiento del rubro de la seguridad social, con lo cual se buscaba que trajera consigo mejores condiciones laborales para toda la población.

Una problemática evidenciada en cuanto al concepto de salarios era la relacionada con los salarios vencidos, los cuales se refieren a aquellos generados en la fecha de despido del trabajador y un período máximo de doce meses; antes de la reforma, no estaba estipulado un límite de fecha, por lo que existía una mala administración de ellos (González, 2013). Esta situación llevó a que se replanteara el mecanismo para calcular estos salarios, con el fin de llevar mayor evidencia y orden en la cobranza por parte de los empleados.

Con el propósito de incentivar y mejorar el poder adquisitivo de los trabajadores, se obligó a los empleadores a afiliar al Infonacot (Instituto del Fondo Nacional para el Consumo de los Trabajadores) a los empleados para que estos pudieran obtener créditos con bajos intereses (Infonacot, 2012).

Otro aspecto que es importante mencionar lo destaca la Secretaría de Gobernación de México (2013), que reitera la formalización de múltiples empleos, entre ellos trabajos a domicilio, actividades realizadas a distancia, entre otras. Del mismo modo, se establecieron mayores capacitaciones con el fin de lograr mayor productividad y se incentivó a los empleados a tener mejores puestos si se capacitan más, para demostrar mejores aptitudes y mayor productividad. No obstante, es importante resaltar que se llevó a cabo una reestructuración de las capacitaciones que permiten generar mayor capital humano al formar individuos más productivos y que puedan tener mayores contribuciones.

Sumado a lo anterior, se comenzó a instaurar un monto mínimo de pago por hora en diversos empleos y se estableció que los salarios podían depositarse por medio de tarjetas de débito, cuentas bancarias y otros medios electrónicos (González, 2013). A su vez, esto tenía por objetivo fortalecer el aparato financiero y mejorar las condiciones de los 
empleados al poseer mayor capacidad adquisitiva, junto con mayor seguridad proporcionada por las entidades financieras.

De igual modo, según la Secretaría de Gobernación de México (2013), la reforma reconoce y permite a los trabajadores realizar actividades fuera del trabajo principal con el propósito de tener mayores ingresos. En cuanto a la infraestructura de los lugares laborales que presenten peligro para los trabajadores, se debe realizar una serie de modificaciones o, de lo contrario, el establecimiento podrá ser cerrado; también se podrán cobrar cuantiosas sanciones, ya que la reforma incrementó sus montos.

\subsection{Efectos de las reformas de México y Colombia sobre sus economías}

Ante el panorama que se pudo desarrollar anteriormente, es posible afirmar que las reformas laborales tanto de México como de Colombia tienen por objetivo mejorar las condiciones de empleo y auspiciar un mejor entorno económico que permita que las personas pertenecientes a cada país puedan disfrutar de mejor calidad de vida. Sin embargo, por un lado, está lo que debería suceder y, por el otro, la realidad que actualmente viven estos países.

Para analizar cuáles han sido las consecuencias de las reformas en las economías y en el nivel de desempleo de ambos países, se procederá a indagar la evolución de las principales variables macroeconómicas, a saber: el crecimiento del PIB (\% anual), la evolución de la tasa de desempleo y los índices de productividad de cada país, para así, al final concluir si a partir de los resultados encontrados en estas variables, las condiciones laborales y de vida de México y Colombia han mejorado.

\section{Crecimiento del PIB como porcentaje anual}

En análisis de este indicador se presenta como uno de los más importantes debido a que todas las políticas y reformas que sean impuestas en un país tienen consecuencias, ya sean relevantes o no, en la evolución de su PIB. Este caso no es la excepción, ya que, como se verá en el siguiente gráfico, los resultados fueron positivos para Colombia a partir del año 2002.

A lo largo del tiempo, Colombia ha afrontado diversas crisis económicas que definitivamente han afectado la evolución de su PIB; sin embargo, también ha mostrado altas tasas de crecimiento que permiten definir su ciclo económico. Como se puede observar en la figural, se destacan tres momentos importantes: un crecimiento del PIB por encima del $8 \%$ en 1977 y dos crisis que tuvieron un impacto significativo, una en 1998 y la que se encuentra en el período de estudio, es decir, la crisis de 2008. Sin embargo, a pesar de todos estos acontecimientos, en 2002, después de venir de una fase de depresión, 
el porcentaje empezó a crecer y se vio nuevamente afectado por la crisis de 2008, pero hacia el año 2010, el PIB recuperó su fuerza y logró mantenerse entre el 3 \% y 5.5 \%.

Figura 1. Crecimiento del PIB (\% anual) para Colombia

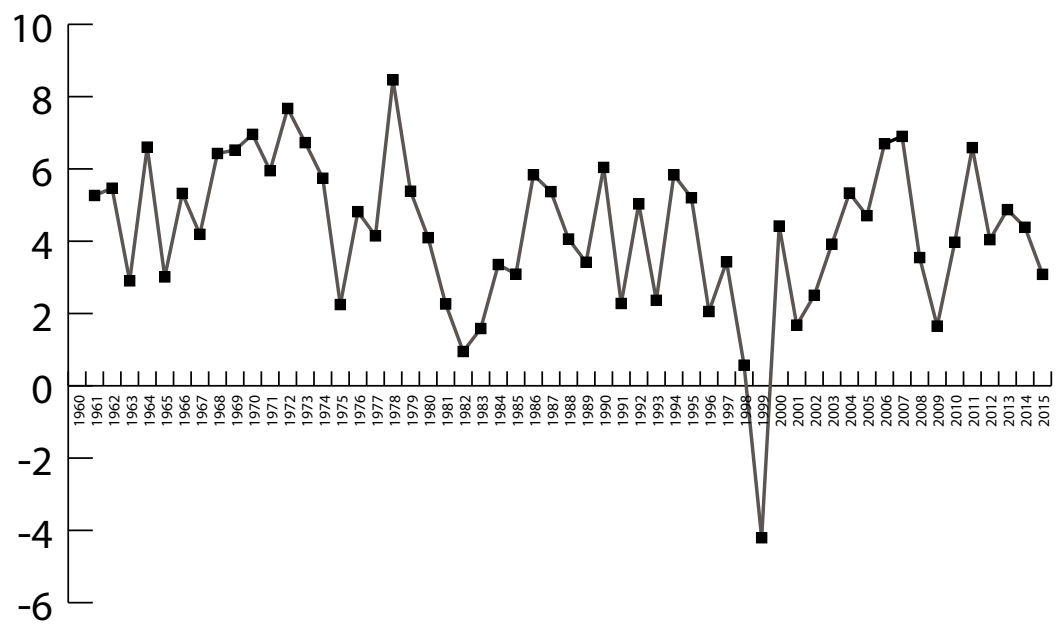

Fuente: elaboración propia con base en datos del Banco Mundial.

Por lo tanto, se puede inferir que, en términos de crecimiento del PIB, al verse después del año 2002 con un comportamiento positivo - a excepción del año 2008-, posiblemente se hayan aumentado la productividad y la demanda agregada, lo que significaría que las personas obtuvieron mayores ingresos para adquirir bienes y servicios. La evolución del PIB (\% anual) se presenta a continuación en la figura 2.

Figura 2. Crecimiento del PIB (\% anual) para México

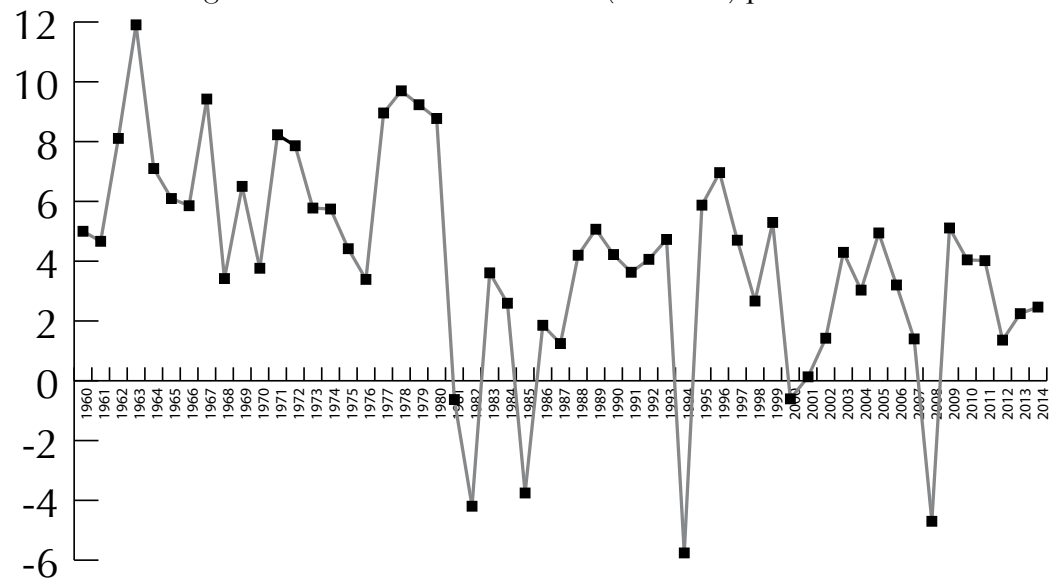

Fuente: elaboración propia con base en datos del Banco Mundial. 
El comportamiento de esta variable para México tiene grandes diferencias en comparación con Colombia en lo que respecta a las diferentes crisis que se han presentado a lo largo de los años. En la figura 2 se puede percibir que este país ha tenido más crisis que Colombia y que estas se han notado más graves. Hay cuatro momentos principales en los que el crecimiento del PIB (\% anual) se ha visto incluso llegar a niveles de evolución negativa y en los que la crisis del año 2008 afectó mucho más. Adicionalmente, se puede encontrar que antes del año 2012, los niveles de crecimiento estaban incluso más altos, pero luego de este año llegó a una breve desaceleración.

Gracias la información que provee la figura 2, y en relación con la reforma de México, se puede inferir que, en lo que respecta al crecimiento económico, no ha tenido mayor impacto, aunque así se muestra un aumento después de 2012, pero incluso si se compara con el año 2010, parece aún más bajo, de hecho, no alcanza a posicionarse más allá del $3.5 \%$.

Tanto para Colombia como para México, los datos recolectados de la evolución del PIB (\% anual) demuestran un crecimiento de este indicador después de aplicada la reforma laboral en cada una de ellos. Según un estudio realizado por Amarante, Arim y Santamaría (2005), las estimaciones que estos investigadores realizaron dieron como resultado que la reforma laboral para Colombia había tenido efectos positivos sobre las horas trabajadas y las remuneraciones. Lo anterior posiblemente confirma el hecho de que la figura 1 haya mostrado un comportamiento creciente desde el año 2002, con excepción del año 2008, debido al posible incremento de la demanda agregada por el aumento de las remuneraciones.

Por su parte, México, a pesar de haber mostrado un comportamiento creciente después de 2012, en comparación con años anteriores, muestra un comportamiento desacelerado que permite deducir que los resultados de las reformas no han sido del todo positivos. Según la investigación de Bensusán (2013), la reforma laboral sí ha cumplido con el objetivo de mejorar los derechos de los trabajadores debido al aumento de las sanciones. Sin embargo, en lo que respecta a estabilidad laboral y a mejores salarios, los resultados no son favorables. La anterior puede ser una de las causas por las cuales no se vio una aceleración significativa en el crecimiento del PIB, pues si una reforma no brinda a los trabajadores la oportunidad de tener mejores salarios o incluso un trabajo estable, es muy dificil que estos puedan acceder con facilidad a los bienes y servicios que necesitan, lo que a su vez estaría generando falta de oportunidades y desigualdad.

\section{Desempleo}

El desempleo es una de las problemáticas más importantes que se encuentran alrededor del mundo y tiene una especial atención, puesto que afectan a todos los países, sin importar el nivel económico en el que se encuentren, incluso a pesar de que las tasas 
son más altas en unos países que en otros. Por lo tanto, las reformas laborales que se aplican en los países, al desenvolverse en el mercado laboral, afecta directamente sobre la variable del desempleo. Es por esta razón que la evolución del desempleo tanto para Colombia como para México es importante para corroborar si los efectos de las reformas económicas fueron positivos. A continuación, en la figura 3 se muestra la evolución del desempleo en Colombia.

Figura 3. Desempleo para Colombia (\% anual)

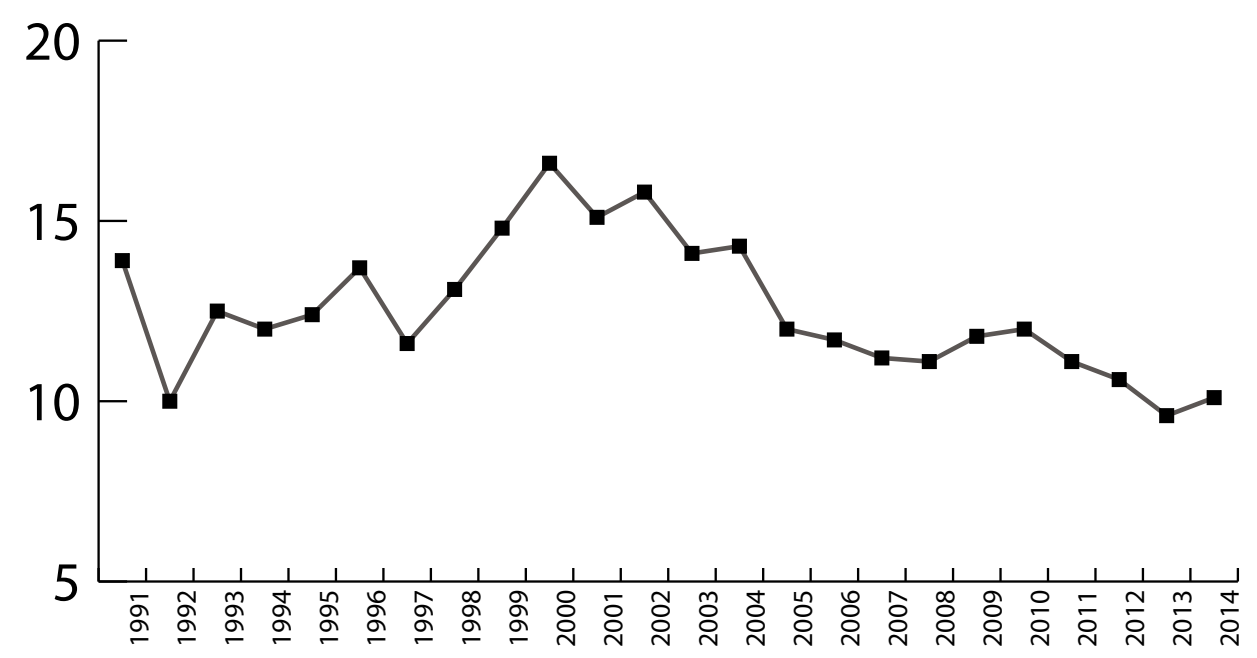

Fuente: elaboración propia con base en datos del Banco Mundial.

Gracias a los resultados que la figura 3 proporciona, es posible afirmar que Colombia ha tenido un problema de desempleo significativamente agudo, puesto que las tasas de desempleo se han fijado hasta por encima del $16 \%$, llegando actualmente a estar por encima del $10 \%$. Desde el año 2002 y hasta el año 2013, se destaca una tendencia a la disminución de esta tasa, sin embargo, a pesar de esta situación, las tasas siguen estando muy altas, lo que perjudica principalmente a la fuerza laboral de país y, por ende, su productividad y competitividad. Según Henao y Rojas (1999), la tasa de desempleo es quizás el indicador más importante para medir la efectividad de una política económica o una reforma, no obstante, los autores afirman que el desempleo, además de lo anterior, refleja el acierto o el desacierto de los programas educativos y de capacitación de la fuerza laboral en términos de cobertura y calidad. Por lo tanto, según la visión de los autores mencionados anteriormente, las altas tasas de desempleo de Colombia están reflejando que, a pesar de que las cifras del PIB están aumentando, la situación laboral de país no es la esperada. Ahora se proseguirá a analizar la misma situación para México. 
Figura 4. Desempleo para México (\% anual)

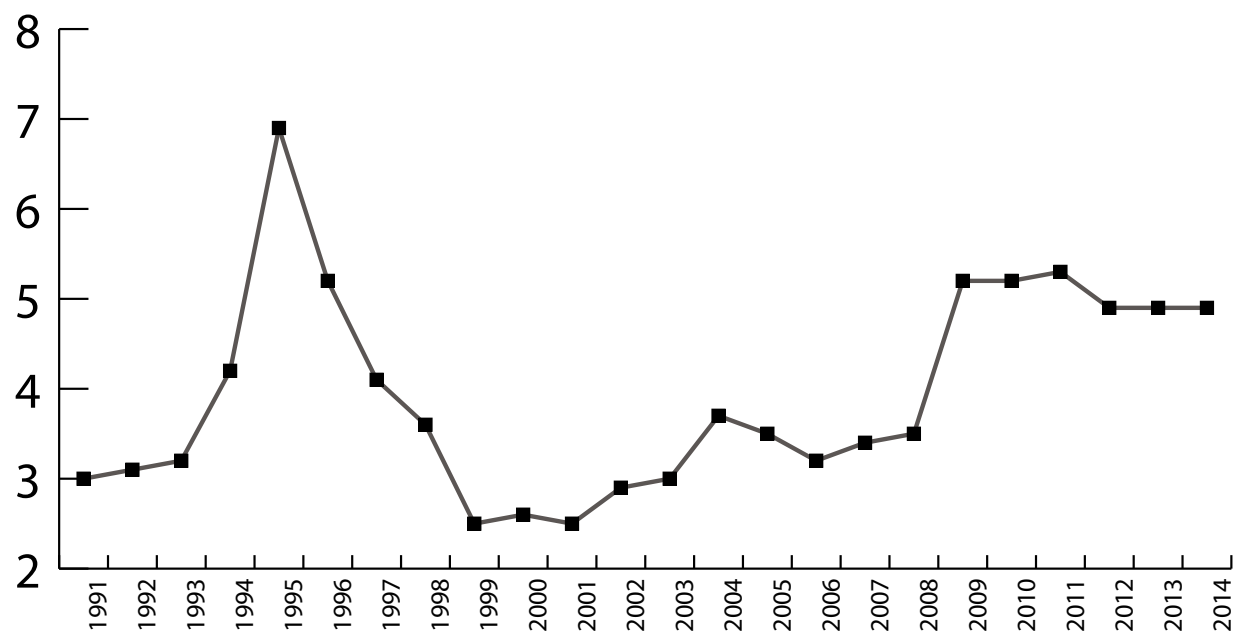

Fuente: elaboración propia con base en datos del Banco Mundial.

La evolución de la variable desempleo para México tiene un componente que la hace muy diferente a la de Colombia, y es que en este país el desempleo, a lo largo del tiempo, ha tenido valores relativamente bajos a pesar de que desde el año 2000 ha mostrado tendencia a aumentar. Vale la pena destacar que se había posicionado entre $3 \%$ y $5 \%$, pero luego del año 2012 se puede observar que comenzó a tener un comportamiento constante hasta 2014. Si se toma en cuenta el hecho de que esto está ocurriendo, es posible concluir que la reforma laboral reflejada en esta variable aportó significativamente a la estabilidad de los empleos y a la vez de los salarios.

Sin embargo, la situación que se acaba de mencionar no necesariamente representa algo bueno para la economía o para las personas en general, ya que es posible que la reforma para México no haya contribuido a la mejora de las condiciones laborales de las personas. Adicionalmente, si realmente la reforma no contribuye a mejorar y aumentar la cantidad de empleos, la situación respecto a los ingresos de las personas tampoco tendrá un cambio positivo y a largo plazo puede que el crecimiento de la economía se vea afectado. De hecho, un informe de la Universidad Nacional Autónoma de México (2013) afirma que, luego de un año de impuesta una reforma que tenía por objetivo la generación de más empleos, el efecto que se encontró fue mayor informalidad y precariedad en el mercado laboral.

Además, luego de haber analizado la evolución del PIB de México y concluir que era una economía con crisis significativas, las cifran presentadas en la figura 4 se muestran sospechosas. Puede que en este país se estén teniendo en cuenta a los empleados informales como personas empleadas normales, sin embargo, esta resultaría ser una situación 
crítica, puesto que significaría que hay muchas más personas sin las condiciones laborales mínimas de las que muestran los datos. En México solo se tiene en cuenta la población desocupada, es decir, que aquellas personas que han estado buscando trabajo, pero que no lo han encontrado y que, por ende, desistieron de la actividad, son eximidas; en otras palabras, en México se intercambia la tasa de desempleo por la tasa de desocupación, lo que hace que esta cifra disminuya significativamente (Universidad Nacional Autónoma de México, 2013).

\section{Productividad}

Analizar la productividad y la eficiencia del uso de los recursos de un país es relevante para evaluar la evolución de un país en términos de crecimiento económico y sostenibilidad. Sin embargo, al hablar de las reformas laborales tanto en Colombia como en México, el sector que se ve principalmente afectado es el industrial, además porque es este por el cual se permite medir la producción promedio de un trabajador en un período determinado.

\section{Colombia}

La apuesta para Colombia hecha por el gobierno en términos productivos se sintetiza en la implementación y creación de la Agenda Interna para la Productividad y la Competitividad, que inició en 2004 (Buitrago Mora, 2013). Esta estrategia fue impuesta para Colombia con el fin de mejorar la productividad en términos de cantidad, pero también de calidad. Según la autora, el aumento de las cantidades que se producen en un tiempo implicaría un esfuerzo por aprovechar las ventajas comparativas que posee el país para mejorar el ingreso, disminuir la pobreza y avanzar en condiciones de equidad. Adicional a lo anterior, el papel que juega la reforma laboral en esta situación es relevante debido a que la fuerza laboral debería ser el foco principal para empezar el mejoramiento de la productividad en el país.

No obstante, a pesar de todas las estrategias y de las reformas económicas que se han implementado para mejorar la productividad en el país para así mejorar la competitividad, los resultados que se han obtenido no han sido muy favorables. Según Morales (2014), Colombia ocupó el puesto 66 entre 144 países en términos de productividad en una medición realizada por el Foro Económico Mundial. Este resultado indica que, indiscutiblemente, Colombia no se encuentra en los niveles de productividad esperados.

Una de las razones por las cuales se está generando este problema es quizás la falta de incentivos que tienen los trabajadores. De hecho, Guataquí y García Suaza (2009) afirman que el efecto de la reforma laboral de 2002 fue principalmente intensivo, lo que significa que la jornada promedio de trabajo se extendió, así como las horas extras trabajadas; 
sin embargo, no hubo efectos positivos sobre la calidad de los empleos, puesto que se registran niveles menores de afiliación a pensiones y seguridad social.

Según el panorama anterior, no hubo efectos muy positivos en términos de productividad, principalmente debido a que la reforma laboral afectó a la mano de obra al aumentar la jornada laboral y disminuir la afiliación a seguridad social. Es posible que exista un desgaste de los trabajadores que les impida realizar sus actividades con más eficiencia y contribuir a la mejora de la productividad, esencialmente en sectores como el industrial.

\section{México}

La economía mexicana ha experimentado diversas transformaciones en las tres últimas décadas. Entre los factores decisivos más importantes se encuentran la liberalización económica y la apertura comercial para abrirle campo a un saldo positivo en el desempeño exportador y un cambio en la estructura productiva. Sin embargo, la situación para este país es muy similar a la de Colombia, puesto que su productividad sigue siendo muy baja. Y es que según la CEPAL (2016), la baja productividad de México ha generado una disminución de la expansión económica de este país. Adicionalmente, la heterogeneidad de productividad entre sectores ha logrado impactos sobre el nivel de desigualdad.

La CEPAL ha reconocido la productividad como un medio para lograr un crecimiento económico con igualdad, es decir, a mayor productividad, mayor crecimiento económico y mejor distribución del ingreso (CEPAL, 2016). Es posible contrastar esto con los resultados de la evolución del PIB para México, que, aunque se mostró con una tendencia positiva, su aceleración no se mostró significativa, por lo que se puede concluir que la baja productividad en México ha sido la responsable de la estabilidad del crecimiento económico.

Sin embargo, una de las razones por las cuales existe baja productividad en los países puede deberse a las medidas que recaen sobre el mercado laboral, puesto que es necesario que exista buena calidad en los empleos para así contribuir al buen desempeño de los empleados; en esta medida, la reforma laboral debe cumplir el papel de proveedora de estas condiciones. Bensusán (2013) afirma que la reforma laboral de 2012 partió de una base en la que era necesario mejorar la flexibilización laboral y los índices de competitividad, sin embargo, las prioridades que surgieron de la situación laboral real no fueron suficientemente consideradas en dicha reforma. A pesar de que los derechos de los trabajadores se defendieron, aún se tolera la mala calidad de los empleos (Bensusán, 2013).

En conclusión, la situación que presenta México es muy similar a la de Colombia, pero México se encuentra en una situación en la que su crecimiento económico se ha visto debilitado debido a los bajos índices de productividad. La reforma laboral, como principal promotora de la estabilización del mercado de trabajo y de los salarios, no 
tuvo en cuenta las verdaderas necesidades que tiene este país, puesto que se dejaron de lado problemáticas como el desempleo y la desigualdad, para tratar temas de menor importancia, como la flexibilización laboral, sin tener en cuenta que al aliviar los problemas sociales que acarrea un mercado laboral débil podrían solucionarse con una mayor eficiencia problemas en la flexibilización laboral y, por ende, mejoraría la productividad del país.

\section{Conclusiones}

El análisis de la evolución de las variables macroeconómicas tanto para México como para Colombia, contrastado con la implementación de las reformas laborales para ambos países, permite concluir que, respecto al PIB, luego de los años en los que estas reformas fueron impuestas, se observa una tendencia positiva; sin embargo, la situación es más preocupante para México debido a que a lo largo de los años ha tenido críticas crisis que no ha permitido que el PIB tenga niveles de crecimiento sostenido ni mayores al $5 \%$ anual. Colombia, en cambio, sí ha tenido un crecimiento de su PIB más constante, y a no ser por la crisis de 2008, que afectó a la mayor parte de los países latinos, la evolución de esta variable se habría visto más alentadora.

Por otro lado, en cuanto al desempleo, los resultados son más positivos, puesto que ambos países han mostrado una disminución importante de esta variable; no obstante, cabe resaltar que los índices siguen estando por encima del $10 \%$ para Colombia, lo que perjudica seriamente el bienestar de las personas, ya que sin una fuente de ingresos estable se torna más difícil el acceso a bienes y servicios. Para el caso de México, se muestran niveles muy bajos de desempleo, aunque se tiene en cuenta que ha venido disminuyendo; sin embargo, vale recordar que diversos estudios confirman que los desempleados no se utilizan como base, sino los desocupados, lo que da como resultado la tasa de desocupación, pero no la desempleo, que sería más alta que la anterior.

Por último, la productividad para México y Colombia sigue siendo muy baja, principalmente porque las reformas laborales aplicadas en ambos países no tomaron en cuenta las verdaderas necesidades que tenía el mercado laboral. Por su parte, para Colombia, el cambio más significativo que tuvo la reforma laboral de 2002 fue la ampliación de la jornada laboral y las horas extras trabajadas, además de disminuir significativamente la afiliación al sistema pensional. En el caso de la reforma laboral de México de 2012, el cambio más relevante fue el aumento de los trabajos, pero con propensión a la disminución de su calidad. Cuando los trabajadores no tienen buenas oportunidades laborales, cuando tienen jornadas extensas de trabajo y no pueden acceder de la mejor manera a un sistema de salud, es posible que a largo plazo exista un desgaste de esta mano de obra, lo cual afectará, en primera medida, la productividad 
de cada uno de los sectores, especialmente el industrial y el agro, que son aquellos que requieren mayor cantidad de trabajadores.

Finalmente, las reformas laborales tuvieron efectos en el mercado laboral para ambos países, sin embargo, estos efectos no fueron los que se esperaban ni tampoco fueron aquellos que solucionarían las problemáticas que los países han sufrido, es por esta razón que la evolución de las variables macroeconómicas, como el PIB, no crecieron de una manera notoria. La baja productividad de México y Colombia sí puede ser una consecuencia de la ineficiencia de las reformas laborales en cuanto a bienestar de los trabajadores, sin incentivos y sin salarios dignos; el desgaste de estos trabajadores afectó, sin duda, la productividad, y a mediano plazo, el crecimiento económico.

\section{Referencias}

Alcaldía de Bogotá. (27 de diciembre de 2002). Ley 789 de 2002 nivel nacional. Por la cual se dictan normas para apoyar el empleo y ampliar la protección social y se modifican algunos artículos del Código Sustantivo de Trabajo. Diario Oficial 45.046 del 27 de diciembre de 2002. Recuperado de goo.gl/tW0CMP

Álvarez Gómez, J. y Alonso González, Á. (2005). Nociones de crecimiento y desarrollo económico. Revista Galega de Economía, 5(2), 1-10. Recuperado de goo.gl/QVeIil

Amarente, V., Arim, R. y Santamaría, M. (septiembre de 2005). Los efectos de la reforma laboral de 2002 en el mercado laboral colombiano. Perfil de Coyuntura Económica, 6, 67-82. Recuperado de goo.gl/HjI2c4

Arango, L. y Hamann, F. (2012). El mercado de trabajo en Colombia. Hechos, tendencias e instituciones. Bogotá: Banco de la República. Recuperado de goo.gl/O3dY3y

Banco de la República. (2016). Desempleo. Recuperado de http://www.banrepcultural. org/blaavirtual/ayudadetareas/economia/desempleo

Bensusán, G. (2013). Reforma laboral, desarrollo incluyente e igualdad en México (Serie Estudios y Perspectivas n.o 143) México: Naciones Unidas - CEPAL. Recuperado de goo. gl/6PEJzE

Buitrago Mora, D. M. (2013). Evolución de la economía colombiana en el período 2002-2010. Bogotá: BID.

Calderón, M. (13 de febrero de 2013). Reforma laboral: régimen de subcontratación. La Razón de México. Recuperado de goo.gl/FZpPu3 
CEPAL. (2016). Productividady brechas estructurales en México. Ciudad de México: Naciones Unidas. Recuperado de goo.gl/hLCLMt

Congreso de los Estados Unidos Mexicanos. (13 de noviembre de 2012). Ley Federal del Trabajo. Decreto por el que se reforman, adicionan y derogan diversas disposiciones de la Ley Federal del Trabajo. Diario Oficial de la Federación del 30 de noviembre de 2012. Recuperado de goo.gl/16QP0v

Gobierno de la República de México. (2013). Reforma laboral. Recuperado de goo.gl/ P9lykm

González, J. de J. (abril de 2013). Reforma laboral: algunos apuntes para el análisis legislativo (Documento de trabajo n.o 148, CESOP). México D. F.: Centro de Estudios Sociales y de Opinión Pública.

Guataquí, J. y García Suaza, A. F. (2009). Efectos de la reforma laboral: ¿más trabajo y menos empleos? Ensayos sobre Política Económica, 27(60), 46-79. Recuperado de goo. gl/0yWs $3 \mathrm{Y}$

Henao, M. L. y Rojas, N. (1999). La tasa natural de desempleo en Colombia. Coyuntura Económica, 29(3), 79-93. Recuperado de goo.gl/008136

Instituto Peruano de Economía. (2016). Productividad laboral. Recuperado de http://www. ipe.org.pe/content/productividad-laboral

Infonacot. (noviembre de 2012). El Infonacot felicita al H. Congreso de la Unión por la aprobación de la reforma laboral (Comunicado de prensa 39/2012). México: Infonacot. Recuperado de goo.gl/WIbAxN

Macías, J. F., Suescún, P. A. y Cardona, M. (26 de marzo de 2009). La educación para el trabajo de jóvenes en Colombia ¿Mecanismo de inserción laboral y equidad? (Documento n.o 354, Serie Archivos de Economía). Bogotá: Departamento Nacional de Planeación.

Morales, M. (2014). Los colombianos trabajan mucho pero les rinde poco. El Tiempo .

Prosperidad Social. (25 de julio de 2016). ¿Qué es jóvenes en Acción? Recuperado de http:/ / www.prosperidadsocial.gov.co/que/jov/Paginas/Requisitos.aspx

Resico, M. (2011). Introducción a la economía social de mercado (Edición latinoamericana). Buenos Aires: Konrad Adenauer Stiftung. Recuperado de goo.gl/emAQrD

Secretaría de Gobernación de México. (2013). Reforma laboral, derecho del trabajo y justicia social en México. Ciudad de México: SEGOB. Recuperado de goo.gl/k0tunI 
Servicio Nacional de Aprendizaje - SENA. (2014). Acuerdo n.o 4 de 2014. El Consejo Directivo Nacional del Servicio Nacional de Aprendizaje SENA. Recuperado el 16 de octubre de 2016, de http://normograma.sena.edu.co/docs/acuerdo_ sena_0004_2014.htm

Soria, J. M. (marzo de 2015). Alcance de la reforma laboral del 2012 y decisiones legislativas pendientes en la materia. Recuperado de http://www.senado.gob.mx/ibd/content/productos/ci/CI_6_DGIE.pdf

Universidad Nacional Autónoma de México (2013). Reporte de investigación 118. Desempleo y menos paga por más horas de trabajo: Resultado a dos años de la reforma laboral. México: Centro de Análisis Multidisciplinario CAM. Recuperado de http://cam.economia. unam.mx/reporte-de-investigacion-1 18-desempleo-y-menos-paga-por-mas-horasde-trabajo-resultados-dos-anos-de-la-reforma-laboral/

Universidad EAFIT. (2006). Reforma laboral. Boletín 74.

Universidad Externado de Colombia. (2005). Mitos y realidades de la reforma laboral colombiana. La Ley 789 dos años después (Cuadernos de Trabajo n.o 6 del Observatorio del Mercado de Trabajo y la Seguridad Social). Bogotá: Universidad Externado de Colombia.

Vega, M. L. (2006). La reforma laboral: ¿un paso necesario? IUSLabor, 3. Recuperado de goo.gl $/ 327 \mathrm{pbZ}$ 